\title{
Umfrage zum Lehramtsstudium
}

Für die GDM führt Prof. Horst Hischer (Saarbrücken) eine erneute Umfrage bei den mathematischen Fachbereichen über den Stand der Umstellung auf Bachelor/Master bzw. andere Modularisierungsmodelle des Lehramtsstudiums durch.

Er schreibt in seinem Anschreiben an die Vorsitzenden der Fachbereiche:

Liebe Kolleginnen und Kollegen, sehr geehrte Damen und Herren,

die gegenwärtige Situation bei der „Reform“ der Lehrerbildung (,,Bologna-Prozess“) lässt befürchten, dass die neuen Studien- und Prüfungsordnungen - entgegen dem ursprünglich angestrebten Ziel! zu einer Vielfalt unterschiedlicher und nicht kompatibler Modelle der Lehramtsstudiengänge führen (können). Eine fundierte Gesamtübersicht über diese Modellvielfalt gibt es bisher leider nicht, sie ist aber dringend erforderlich.

Der Vorsitzende der Gesellschaft für Didaktik der Mathematik e. V. (GDM), Koll. Hans-Georg Weigand aus Würzburg, hat daher eine entsprechende Umfrage unter den deutschen Hochschulen angeregt, um einen Überblick über diese neuen Struk- turen der Lehrerbildung in Deutschland gewinnen zu können. Er hat mich gebeten, diese Umfrage zu organisieren, und er bittet Sie hiermit um Ihre Mithilfe!

Sie erinnern sich daran, dass wir in Heft I von 2006 einen Fragebogen der DMV zum gleichen Thema hatten, den wir seinerzeit leider nur von 16 Universitäten ausgefüllt zurück bekommen hatten.

Wenn Sie mit uns glauben, dass es wichtig ist, zu diesem Thema möglichst viele Informationen verfügbar zu haben, und an einem mathematischen Fachbereich tätig sind, dann fragen Sie bitte nach, ob die Verantwortlichen Ihres Fachbereichs an der neuen Umfrage bereits teilgenommen haben und veranlassen Sie bitte ggf., dass das noch geschieht.

Die Daten werden durch ein Internetformular (passwortgeschützt) auf der Seite http:// mathematikunterricht.info/BildPol/lehramt/ erhoben, alle Fachbereiche haben das Passwort erhalten. Auf dieser Seite kann auch der aktuelle Stand der Umfrage eingesehen werden.

(RSP) 